\title{
Self-similar dynamics of air film entrained by a solid disk in confined space: A simple prototype of topological transitions
}

\author{
Hana Nakazato, Yuki Yamagishi, and Ko Okumura* \\ Department of Physics and Soft Matter Center, Ochanomizu University, 2-1-1 Otsuka, Bunkyo-ku, \\ Tokyo 112-8610, Japan
}

(Received 8 December 2017; published 17 May 2018)

\begin{abstract}
In hydrodynamic topological transitions, one mass of fluid breaks into two or two merge into one. For example, in honey-drop formation when honey is dripping from a spoon, honey is extended to separate into two masses as the liquid neck bridging them thins down to the micron scale. At the moment when the topology changes due to the breakup, physical observables such as surface curvature locally diverge. Such singular dynamics has widely attracted physicists, revealing universality in self-similar dynamics, which shares much in common with critical phenomena in thermodynamics. Many experimental examples have been found, including an electric spout and vibration-induced jet eruption. However, only a few cases have been physically understood on the basis of equations that govern the singular dynamics and even in such a case the physical understanding is mathematically complicated, inevitably involving delicate numerical calculations. Here we study the breakup of air film entrained by a solid disk into viscous liquid in a confined space, which leads to formation, thinning, and breakup of the neck of air. As a result, we unexpectedly find that equations governing the neck dynamics can be solved analytically by virtue of two remarkable experimental features: Only a single length scale linearly dependent on time remains near the singularity and two universal scaling functions describing the singular neck shape and velocity field are both analytic. The present solvable case would be essential for a better understanding of the singular dynamics and will help reveal the physics of unresolved examples intimately related to daily-life phenomena and diverse practical applications.
\end{abstract}

DOI: 10.1103/PhysRevFluids.3.054004

\section{INTRODUCTION}

The self-similar dynamics in hydrodynamics was already in focus when the dynamics of viscous instability of a moving front was studied [1] and the renormalization group theory in statistical physics, which elucidates universality appearing in critical phenomena in thermodynamics, was recognized worldwide beyond fields [2]. Subsequently, the self-similar dynamics was studied for the breakup of droplets (capillary pinch-off) [3], one typical example of topological transitions. In that seminal paper, the scaling Ansatz, one of the key ideas leading to universality in critical phenomena, was shown to be useful. This paper ignited a surge of publication on the self-similarity in fluid breakup phenomena [4-7].

Thereafter, an analytical solution for the dynamics of droplet coalescence, another typical example of hydrodynamic topological transitions, was published [8]. This was followed by many studies of

\footnotetext{
*okumura@phys.ocha.ac.jp
}

Published by the American Physical Society under the terms of the Creative Commons Attribution 4.0 International license. Further distribution of this work must maintain attribution to the author(s) and the published article's title, journal citation, and DOI. 
the dynamics of droplets and bubbles, such as coalescence [9-12], noncoalescence [13], pinch-off $[14,15]$, and droplet impact onto substrates [16-21], with a rapid technological advance in high-speed imaging and numerical computing. However, since then, the field has been developed rather remotely from the self-similar dynamics, while one of the main foci has been on simple scaling laws [22], which is another key concept in understanding universality in critical phenomena.

To date, many types of singular transitions, which are more general than topological transitions, and associated self-similar dynamics have been found experimentally in hydrodynamics. Such singular transitions often exhibit universality via self-similar dynamics near the singular point, while nonuniversal behavior is observed under certain conditions [23]. Other examples often related to practical applications include the spout of a liquid jet under an electric field [24] (closely related to inkjet printing) and jet eruption under vibration [25]. In addition, the self-similar dynamics was found in droplet coalescence under an electric field [26] (relevant to microfluidic applications). Self-similarities are sometimes identified not only in the dynamics as in the above examples but also in the steady-state flow towards singular transitions, in which the topology is not necessarily changed, as observed in flow-induced air entrainment [27-29] and selective withdrawal [30]; both of these conditions are useful, e.g., for macroscopic [31] and microscopic coatings [32] important for chemical, biological, and medical engineering.

As indicated above, each example of singular transitions is often familiar to everyone and/or important for applications. In fact, our example of fluid breakup is central to diverse processes, involving two-phase flows, emulsification, and drop formation, in industrial, engineering, and scientific realms. Understanding physical laws governing the dynamics is useful for various purposes [33], e.g., (i) industrial production of food such as mayonnaise and cream and of spray systems for painting or planting, (ii) engineering development of microfluidic devices that are promising for applications in chemistry, biochemistry, and material science, and (iii) atmospheric science dealing with the formation of raindrops and thunderstorms.

As for the emergence of universality, singular transitions are analogous to critical phenomena in thermodynamic transitions [34-36]. By identifying characteristic physical quantities and lengths describing the fluid dynamics near the singularity, the properties of the interface and the flow can be understood in terms of a similarity solution near the transition point, which allows a simplified description of the dynamics, leading to a deeper understanding of the phenomena.

However, only a few examples of the above-mentioned singular dynamics have been understood, and even if well understood, the theory is intricate, involving delicate numerical calculations. (In contrast, the universal dynamics associated with hydrodynamic instability has been rather simply understood [1,37].) Because of this, understanding of the singular dynamics is premature. If this were not the case, universalities for the singular dynamics could have been classified, as has been done for critical phenomena.

Here we report an experimental and theoretical study of the appearance of a singularity by air entrainment caused by a solid disk in a confined viscous medium in the Hele-Shaw cell. The remarkable features of the present case are that only a single length scale remains near the singularity to characterize the dynamics and that the length scale simply scales linearly with time. These exceptionally simple characteristics make the theory completely solvable in a perturbative sense, leading to analytic scaling functions and demonstrating the essential physical mechanisms of the singular dynamics in a clear way. Most of previous experimental examples possess more than one remaining length scales near the singularity and at least one of the universal scaling functions is nonanalytic. This is indeed the case for well-understood cases [7] such as the pioneering study associated with the Hele-Shaw cell [3] [see just below Eq. (7)] and another seemingly similar case of the flow-induced air entrainment [27,29] (see Sec. III for details). Accordingly, the present study advances our general understanding of the singular dynamics; it represents an important fundamental example of the singular dynamics, providing insight into unresolved self-similar dynamics such as the electric spout of liquid [24], the selective withdrawal [30], and the electric-field-induced drop coalescence [26], and impacting the study of the dynamics of droplets and bubbles in general. 


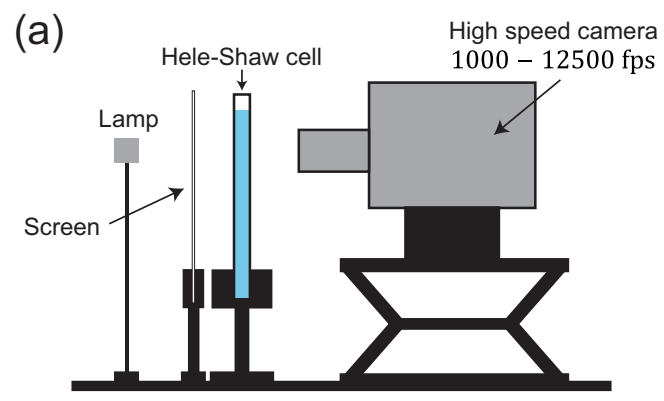

(b)

(c)

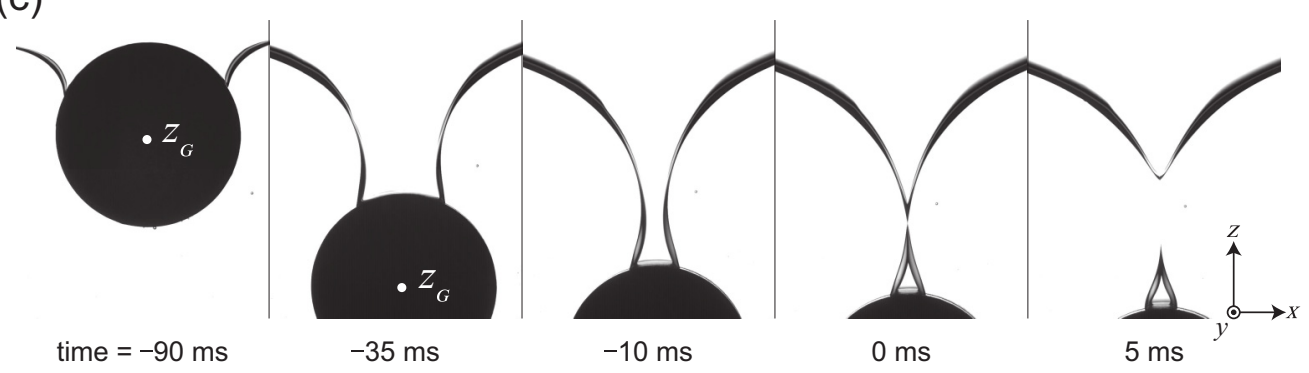

FIG. 1. (a) Experimental setup. (b) Experimental geometry. The disk of radius $R$ with thickness $D_{0}$ falls in the Hele-Shaw cell of thickness $D$ filled with a silicone oil of viscosity $\eta$ and kinematic viscosity $\nu=\eta / \rho$ ( $\rho$ is the density of the oil). Thin oil films of thickness $e$ exist between the surfaces of the disk and cell walls. (c) Overall pinch-off dynamics of air dragged by the disk observed from the front of the cell for $(v, D, R, e)=(100,4,10,0.5)$, where $v$ is given in units of $\mathrm{cS}$ and the others are in $\mathrm{mm}$.

\section{RESULTS}

\section{A. Experiment}

The experimental setup is shown in Fig. 1(a). We create a Hele-Shaw cell with thickness $D$ and fill the cell with a viscous oil [polydimethylsiloxane (PDMS)] with kinematic viscosity $v=\eta / \rho$, where $\eta$ and $\rho$ are the viscosity and density of the oil. We insert a stainless disk [radius $R$, thickness $D_{0}(<D)$, and density $\rho_{s}$ ] at the top of the cell. The bottom of the disk touches the liquid-air interface with zero velocity and the disk starts to go down in the oil phase due to gravity. Since the disk thickness is slightly thinner than the cell thickness $D$, the disk does not directly touch the cell plates and a thin layer of oil is formed between the front cell plate and the front surface of the disk. The thickness of the film $e$ is practically set to the value $e=\left(D-D_{0}\right) / 2$ [Fig. 1(b)]. As a result, the air is dragged by the disk as in Fig. 1(c), forming a singular shape, with details revealed in Figs. 2(a) and 2(b) (see movies 1-4 in [38]), and finally pinches off to cause a topological transition.

The $(x, y, z)$ coordinate system is specified in Fig. 2(c) [see also Fig. 1(c), in which the center of gravity of the disk is denoted by $z_{G}$ ]. For simplicity, the space-time position at the critical moment of pinch-off $(t, x, z)=\left(t_{c}, x_{c}, z_{c}\right)$ is set to the origin of the $(t, x, y)$ coordinate if not specified. The right-hand side of the air-liquid surface is described by $x=h(t, z)$. The minimum of the function $h(t, z)$ as a function of $z$ is defined as $(x, z)=\left(h_{m}, z_{m}\right)$. Under our experimental conditions, the shape of the neck is not axisymmetric and is rather independent of the $y$ coordinate. In fact, as shown in the magnified snapshot in Figs. 2(a) and 2(b), the singular neck shape near the pinch-off point is like a sheet of thickness $2 h_{m}$ and width $\simeq D_{0}$.

Now we discuss experimental details of the experiment. The height and width of Hele-Shaw cells are 12 and $9 \mathrm{~cm}$, respectively. The two plates are separated by a spacer of thickness $D$. To make the thickness of two thin film layers formed on both sides of the disk nearly equal, we attach two small acrylic plates on the inside surfaces of the cell plates near the top of the cell. The thickness 

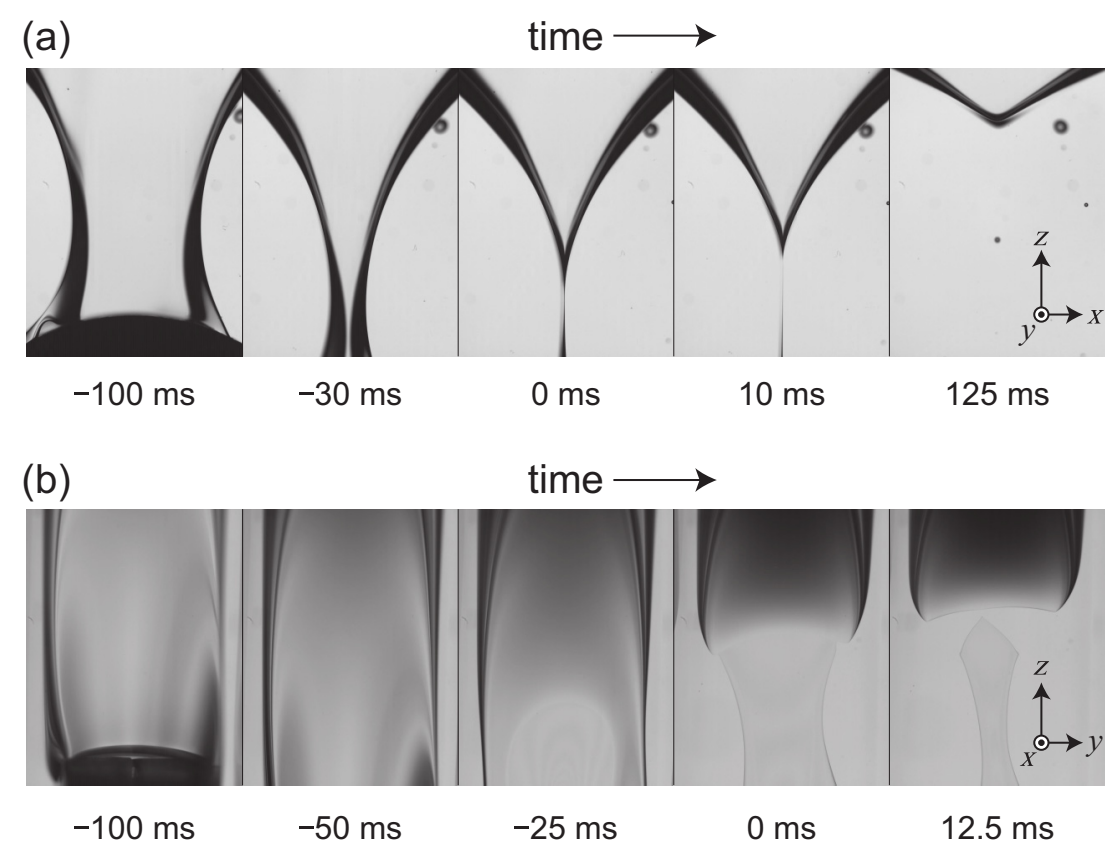

(c)
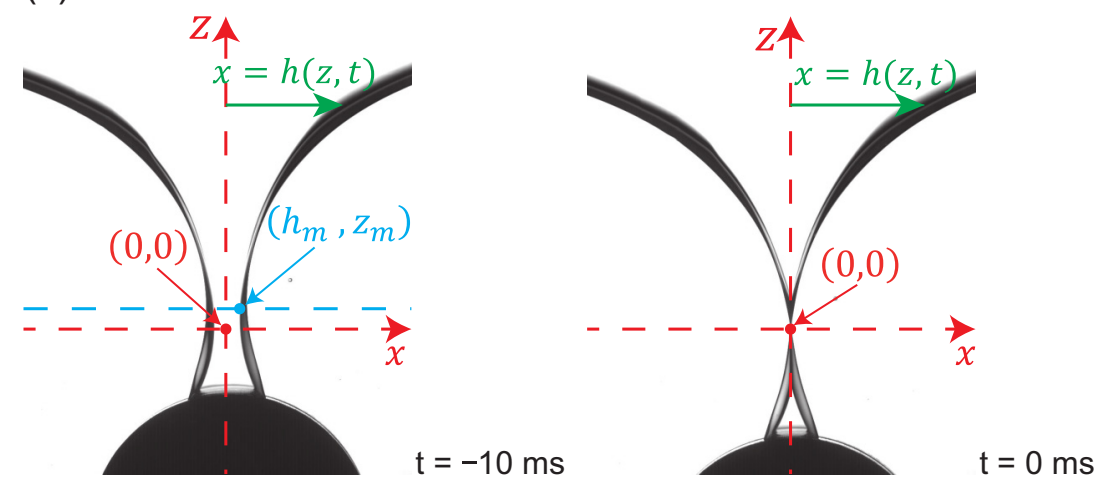

FIG. 2. Pinch-off dynamics observed from (a) the front and (b) the side for $(v, D, R, e)=(500,5,10,0.5)$ with the units specified in Fig. 1. (c) Coordinate system: definitions of geometrical parameters, the minimum neck width $2 h_{m}$, the vertical position of the minimum neck $z_{m}$, and the neck profile $x=h(z, t)$. Snapshots are taken for $(\nu, D, R, e)=(100,3,10,0.5)$.

of the small plates is slightly smaller than $e$ and the two attached plates play the role of a gate for the inserted disk. In the present experiment, we examine different values of $e(=0.2,0.5$, and $1.0 \mathrm{~mm}$ ) and confirm that the results are independent of the value of $e$ in the range. The disk is prewetted by the oil before being dropped into the cell; there is no contact line on the surface of the disk, which removes any effects of the contact angle on the phenomena. When the cell thickness is smaller than the values $(3-5 \mathrm{~mm})$ studied in the present study, the falling velocity of the disk becomes significantly smaller. There seems to be another regime in which the cavity shape near the contact with the disk becomes conelike, unlike the present regime characterized by the sheet formation; this issue is beyond the scope of the present work. We use a high-speed camera (Fastcam SA-X, Photoron) with a macrolens (Micro NIKKOR 60-mm f2.8G ED, Nikon) and analyze images with 
(a)

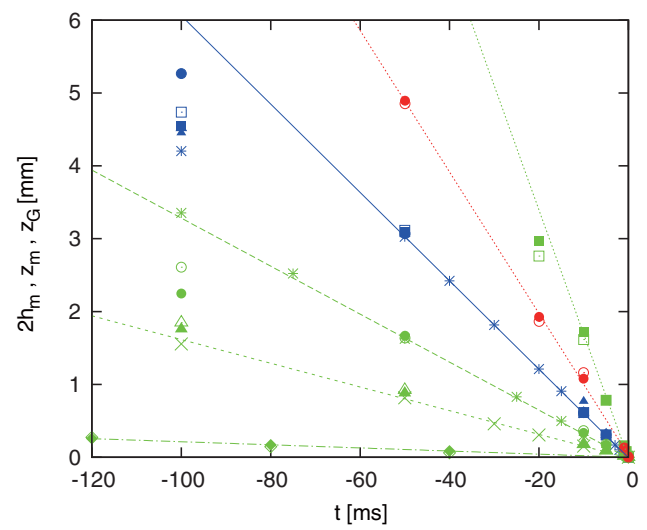

(b)

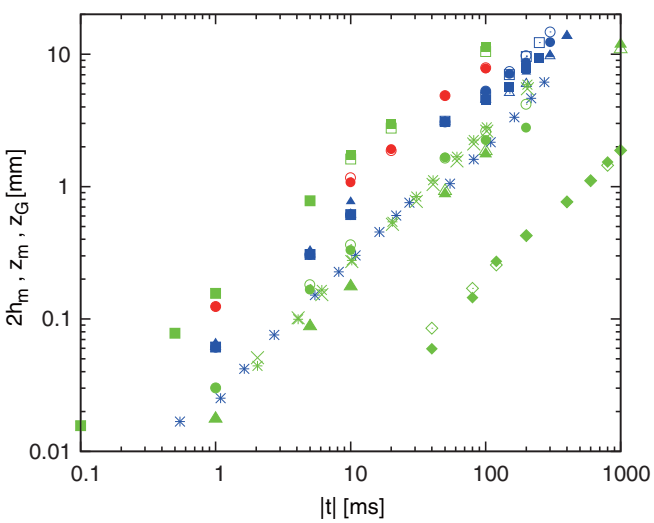

(c)

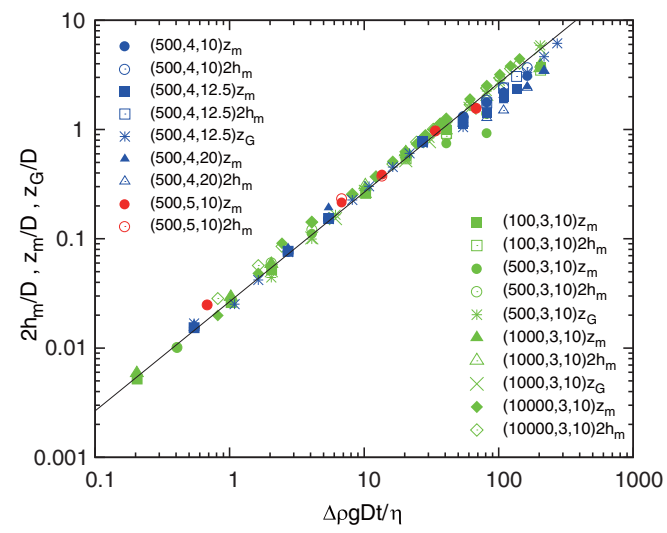

FIG. 3. Plots of the neck width $2 h_{m}$, the vertical position of the neck $z_{m}$, and the center of mass of the disk $z_{G}$ as a function of time $t\left(t\right.$ and $z_{m}$ are measured from the origins $t=t_{c}$ and $z=z_{c}$, respectively, while $z_{G}$ is from $z=z_{G}$ at $\left.t=t_{c}\right)$. The symbols are specified in the plots by the set $(v, D, R)$ as in Figs. 1 and 2. (a) All the data are on a linear-linear scale. (b) The same data as in (a) but on a log-log scale. (c) All the data are collapsed on a single master line with slope one, establishing Eq. (1) with Eq. (2); the best-fitting line gives $k=0.02764 \pm 0.00008$. The dashed lines in (a) are a guide to the eye.

Image J software. The density of the stainless steel (SUS430) is $7.7 \mathrm{~g} / \mathrm{cm}^{3}$, while that of the PDMS depends on viscosity: $0.965 \mathrm{~g} / \mathrm{cm}^{3}$ for $100 \mathrm{cS}, 0.97 \mathrm{~g} / \mathrm{cm}^{3}$ for 500 and $1000 \mathrm{cS}$, and $0.975 \mathrm{~g} / \mathrm{cm}^{3}$ for $10000 \mathrm{cS}$.

\section{B. Dynamics of the neck}

Figures 3(a) and 3(b) show $z_{m}$ and $h_{m}$, together with $z_{G}$, as a function of time for various parameters $\nu, D$, and $R(e=0.5 \mathrm{~mm})$. Figure 3(c) clearly shows the relation

$$
2 h_{m}=z_{m}=z_{G} \equiv l(t),
$$

where $l(t)(t<0)$ is linearly dependent on $t$ :

$$
l(t) \equiv-v_{0} t,
$$


(a)

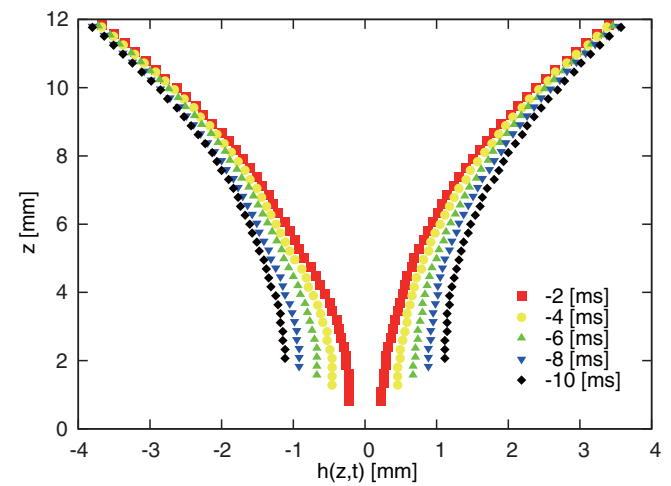

(c)

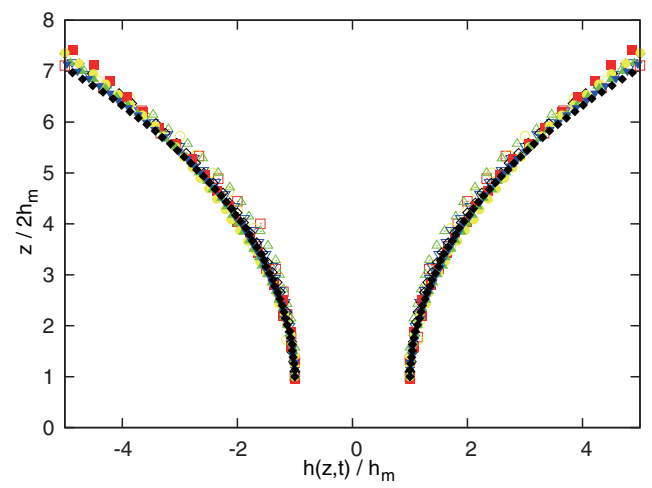

(b)

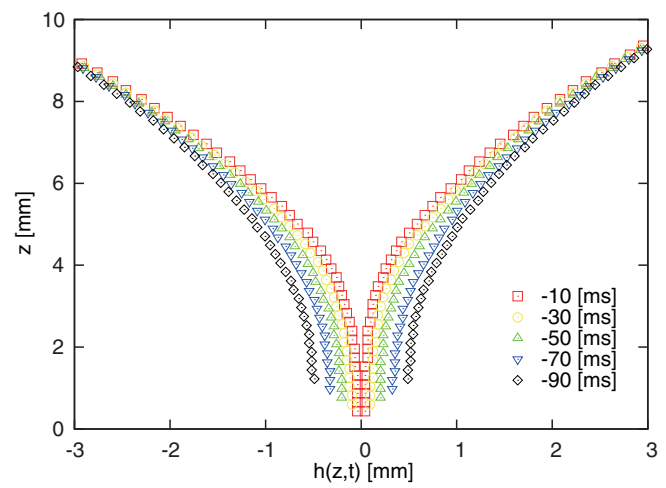

(d)

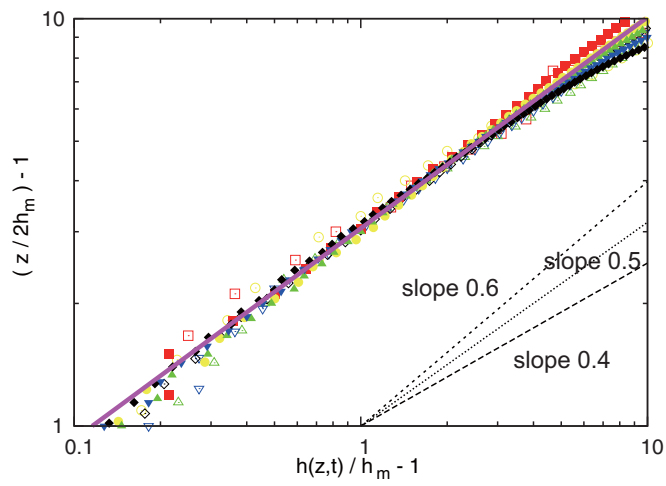

FIG. 4. Self-similar dynamics of the neck shape. The neck profile $h(z, t)$ is shown as a function of $z$ at different times for (a) $(v, D, R, e)=(100,4,12.5,0.5)$ and (b) $(500,3,10,0.5)$ in units of cS and mm. (c) Profiles in (a) and (b) collapsed by rescaling with a single length scale $2 h_{m}=z_{m}=l(t)$. (d) Right branches of the collapsed neck shape in (c) on a log-log scale, establishing Eq. (4) with the best-fitting line giving $a=3.1415 \pm 0.011$.

where $v_{0}=k \frac{\Delta \rho g D^{2}}{\eta}$ with $k=0.02764 \pm 0.00008$, which is close to $1 / 12 \pi \simeq 0.0265$, and $\Delta \rho=$ $\rho_{s}-\rho$ (see Sec. III for the expression for $v_{0}$ ).

The plots in Figs. 4(a)-4(c) demonstrate, for two different parameter sets, that the neck profile $h(t, z)$ has the following scaling form, demonstrating a self-similar dynamics with the single length scale $l(t)$ :

$$
2 h(t, z) / l(t)=H[z / l(t)] .
$$

Figure 4(d) shows that the data agree quite well with the expression

$$
H(\xi)=1+a(\xi-1)^{2},
$$

with $\xi=z / l$ and $a=3.1415 \pm 0.011$, which is close to $\pi$. As stated above, the remanence of only a single length scale near singularity is an exceptionally simple case, similar to the coalescence case [26], for which physical understanding is lacking.

Further details are discussed below. Since the liquid completely wets the cell plates, we measure the inner edge of the neck image as $2 h$. In Figs. 3(a) and 3(b) the dashed lines (the guide to the eye) are drawn by fitting the first three data points (excluding the data at $t=0$ ) for $z_{G}$ (or $z_{m}$ if $z_{G}$ is not available). In Figs. 4(c) and 4(d) the average of the left and right branches is used for the analysis to reduce the experimental error. 


\section{Theory}

As shown below, the existence of the self-similar solution in Eq. (3) with its asymptotic form in Eq. (4) and the linear dependence of $l(t)$ on $t$ in Eq. (2) can be explained by considering the air flow near the neck. Below, the physically most important component of the flow, i.e., the $z$ direction $v_{z}$, is denoted by $v$. Considering that a sheet of air with thickness $\simeq 2 h$ (and width in the $y$ direction $\simeq D_{0}$ slightly smaller than $D$ ) is formed near the neck, as can be seen in Figs. 2(a) and 2(b), it is reasonable to assume that $v$ is independent of $y$ near $y=0$ because $h \ll D(y=0$ corresponds to the central position between the two walls separated by the distance $D$ ). We can further assume that $v$ is independent of $x$, i.e., $v=v_{z}(t, z)$, near the pinch-off point. This is because the narrowest point of the neck located at $z=z_{m}=l(t)=-v_{0} t$ moves downward with the velocity $v_{0}(>0)$ and thus the liquid-air interface drags down air at the interface, which makes a plug flow inside the air neck, since the Reynolds number $\operatorname{Re}=\rho_{a} v_{0} h / \eta_{a}$ becomes infinitely small towards the singular point $\left(\rho_{a} \simeq 1 \mathrm{~kg} / \mathrm{m}^{3}\right.$ and $\eta_{a} \simeq 10^{-5} \mathrm{~Pa}$ s are the density and viscosity of air, respectively, and $\operatorname{Re}<0.1$ for $h<1 \mathrm{~mm}$ since $v_{0}<10^{-3} \mathrm{~m} / \mathrm{s}$ ). Now that we have justified the independence of $v$ from $x$ and $y$ near the singularity, we obtain the condition

$$
v\left(t, z=z_{m}\right)=-v_{0}
$$

and the equation of continuity

$$
\frac{\partial h}{\partial t}+\frac{\partial h v}{\partial z}=0
$$

Equation (6) can also be justified in the following manner. The pressure value at the neck is almost precisely equal to that of the atmospheric pressure $p_{0} \simeq 10^{5} \mathrm{~Pa}$, which implies $\partial \rho_{a} / \partial t=0$ (with the air density $\rho_{a}$ ) even for a thin neck. This is because Laplace's pressure jump $\Delta p$ is negligible compared with $p_{0}$ as long as the radius of curvature of the air-liquid interface is larger than $0.01 \mathrm{~mm}$, which is the present case; since the neck forms a sheet, the radius of curvature is independent of the width of the neck. This is in contrast to the axisymmetric case, in which the radius of curvature scales as the thin neck width and thus the pressure jump becomes significant at the neck.

The dynamics of $h$ and $v$ coupled in Eq. (6) is completely specified by introducing one more equation: the $z$ component of the Navier-Stokes equation in the small- $h$ limit, in which viscosity dominates inertia (note that $\partial_{x} v_{z}=0$ as before):

$$
\eta_{a} \frac{\partial^{2} v}{\partial z^{2}}=\frac{\partial \Delta p}{\partial z}
$$

where $\Delta p=-\gamma \frac{\partial^{2} h}{\partial z^{2}}$, with $\gamma$ the surface tension of the liquid. In the pioneering study in [3], the left-hand side of this equation is replaced by an averaged Poiseuille flow independent of $z$, which unexpectedly leads to a much more complicated scenario for the appearance of universality.

Equation (7) can also be justified in the following manner, i.e., we can confirm retrospectively that the dominating terms in the $z$ component of the Navier-Stokes equation are indeed expressed as in Eq. (7) near the singularity. From the experimental results, near the singular point, we expect characteristic scales in the $x$ and $z$ directions $l_{x}$ and $l_{z}$ and a characteristic time scale $\tau$ can be given by $l_{x}=l_{z}=\varepsilon l_{0}$ and $\tau=\varepsilon t_{0}$ by introducing a small dimensionless parameter $\varepsilon$ and units of length and time $l_{0}$ and $t_{0}$, respectively, which is explained as follows. Since the experiment suggests that there is only a single length scale near the singularity, we can set $l_{x}=l_{y}=\varepsilon l_{0}$. The velocity in the $z$ direction is characterized by $v_{0}$, a finite value independent of $\varepsilon$. This implies that the characteristic time scale for the problem is given by $\tau=\varepsilon t_{0}$ because $v_{0} \simeq l_{x} / \tau \simeq l_{y} / \tau$ is independent of $\varepsilon$. We can now estimate the order of each term in the $z$ component of the Navier-Stokes equation. The inertial terms $\rho_{a}\left(\partial_{t} v_{z}+v_{x} \partial_{x} v_{z}+v_{z} \partial_{z} v_{z}\right)$ scale as $\varepsilon^{-1}$, the gravitational term $\rho_{a} g$ scales as $\varepsilon^{0}$, and the terms remaining in Eq. (7) all scale as $\varepsilon^{-2}$, which verifies that Eq. (7) properly collects relevant leading-order terms near the singularity where $\varepsilon \approx 0$. 
We employ the scaling Ansätze [3,4,6,7,23,39]

$$
2 h(t, z)=z_{0} \tilde{t}^{\alpha} H\left(\tilde{z} / \tilde{t}^{\beta}\right), \quad v(t, z)=-v_{0}^{\prime} \tilde{t}^{\gamma} V\left(\tilde{z} / \tilde{t}^{\delta}\right),
$$

introducing dimensionless scaling functions $H$ and $V$ along with the dimensionless variables $\tilde{z}=\left(z-z_{c}\right) / z_{0}=z / z_{0}$ and $\tilde{t}=v_{0}^{\prime}\left(t_{c}-t\right) / z_{0}=-v_{0}^{\prime} t / z_{0}$, where $z_{0}$ and $v_{0}^{\prime}$ are arbitrary length and velocity scales, respectively, and substitute them into Eqs. (6) and (7). [Note in Eq. (8) that $\gamma$ denotes a dimensionless exponent, not the surface tension.] We here require that the Ansätze are relevant for these equations near the singularity where $\tilde{t} \approx 0$ (i.e., equating all the exponents for the variable $\tilde{t}$ ) to obtain $\alpha=\beta=\delta=1$ and $\gamma=0$. This set reproduces (with the identification $v_{0}=v_{0}^{\prime}$ ) the scaling form in Eq. (3) and reveals another scaling structure

$$
v(t, z)=-v_{0} V[z / l(t)] .
$$

In other words, the present analysis concludes that there is only a single length scale $l(t)$ near the singularity as observed in experiment, which linearly scales with $t$.

Substitution of the scaling forms in Eqs. (3) and (9), thus obtained theoretically, into Eq. (6) makes the variables $t$ and $\xi=z / l$ separate: The original partial differential equation can be changed into a set of ordinary differential equations [Eq. (10) confirms the linear $t$ dependence of $l(t)$; see the next paragraph for details]

$$
\begin{gathered}
d l / d t=-v_{0}, \\
(H V)^{\prime}=\xi H^{\prime}-H .
\end{gathered}
$$

Here $H$ and $V$ are a function of $\xi$ and the prime indicates the derivative with respect to $\xi$. The same substitution into Eq. (7) results in the expression

$$
V^{\prime \prime}=\lambda H^{\prime \prime \prime},
$$

with $\lambda=-\gamma /\left(2 \eta v_{0}\right)$. Equations (11) and (12) should satisfy the boundary conditions [see Fig. 4 for the first two and Eq. (5) for the last]

$$
H(1)=1, \quad H^{\prime}(1)=0, \quad V(1)=1 .
$$

Instead of starting from the general Ansätze in Eq. (8), we can start directly from the scaling Ansatz in Eqs. (3) and (9), motivated by experimental results, without assuming the linear $t$ dependence of $l(t)$ (accepting the existence of a single length scale near the singularity as an experimental fact). From this standpoint, we can show from Eq. (10) that $l(t)$ should be linearly dependent on $t$ and we can explain the asymptotic form of the self-similar shape in Eq. (4).

To our surprise, the boundary problem defined by the two ordinary differential equations for $H$ and $V$ in Eqs. (11) and (12) with the boundary conditions in Eq. (13) can be solved perturbatively as explained in the next paragraph. By assuming the series expansion $H(\xi)=a_{0}+a_{1} \zeta+a_{2} \zeta^{2}+\cdots$ with $\zeta=\xi-1$, we can show $a_{0}=1, a_{1}=0, a_{2}=3 \lambda a_{4}$, and $a_{3}=0, \ldots$. Namely, we obtain, for $\zeta \ll 1$

$$
H(\xi)=1+a_{2} \zeta^{2}+\cdots, \quad V(\xi)=1-\zeta+\cdots,
$$

which explains the experimental result including Eq. (4). Note that we can show $a_{n}=0$ for odd $n$ and we can derive an expression for $a_{n}$ as a function of $a_{m}$ (with $m<n$ ) for even $n$ up to any desired order; the present boundary problem can be solved completely in a perturbative sense. In this example of the hydrodynamic singular transition the scaling functions for the shape and velocity $(H$ and $V$ ) are both analytic; in contrast, in all the previously known examples, at least one of the scaling functions is nonanalytic.

Now we derive Eq. (14). Equation (11) can be rearranged as

$$
\left(1+V^{\prime}\right) H=(\xi-V) H^{\prime},
$$


from which we obtain

$$
\frac{d H}{H}=f(\xi) d \xi
$$

with $f(\xi)=\frac{1+V^{\prime}}{\xi-V}$. By using the boundary condition $H(1)=1$, we get

$$
H(\xi)=e^{F(\xi)},
$$

with $F(\xi)=\int_{1}^{\xi} f(\xi) d \xi$. Since the definition of $f(\xi)$ in Eq. (16) can be recast into the form

$$
V^{\prime}+f(\xi) V=\xi f(\xi)-1,
$$

this first-order linear differential equation for $V$ can be solved as

$$
V(\xi)=e^{-F(\xi)}\left[\int_{1}^{\xi} d \tilde{\xi} e^{F(\tilde{\xi})}\{\tilde{\xi} f(\tilde{\xi})-1\}+1\right],
$$

which satisfies $V(1)=1$. Using Eq. (17), we get

$$
\begin{aligned}
V(\xi) & =\frac{1}{H}\left[\int_{1}^{\xi} d \tilde{\xi} H\left\{\xi \frac{H^{\prime}}{H}-1\right\}+1\right] \\
& =\xi-2 \int_{1}^{\xi} d \tilde{\xi} H(\tilde{\xi}) / H(\xi) .
\end{aligned}
$$

From the conditions $H(1)=1$ and $H^{\prime}(1)=0$ we expand $H(\xi)$ as

$$
H(\xi)=1+a_{2} \zeta^{2}+a_{3} \zeta^{3}+a_{4} \zeta^{4}+\cdots,
$$

with $\zeta=\xi-1$. From this we obtain

$$
\begin{aligned}
V(\xi) & =\xi-2 \zeta \frac{1+a_{2} \zeta^{2} / 3+a_{3} \zeta^{3} / 4+a_{4} \zeta^{4} / 5+\cdots}{1+a_{2} \zeta^{2}+a_{3} \zeta^{3}+a_{4} \zeta^{4}+\cdots} \\
& =1-\zeta+2 \zeta\left[2 a_{2} \zeta^{2} / 3+3 a_{3} \zeta^{3} / 4-\left(2 a_{2}^{2} / 3-4 a_{4} / 5\right) \zeta^{4}+\cdots\right]
\end{aligned}
$$

Substituting Eqs. (22) and (23) into Eq. (12), we can determine $a_{n}$ perturbatively as a function of $a_{m}$ (with $m<n$ ) to obtain Eq. (14).

\section{DISCUSSION}

Among the previous studies of singular transitions, the present problem may be very similar to air entrainment induced by flow [27,29] in that (i) this singularity occurs for air surrounded by viscous liquid and (ii) a thin sheet of air is formed so that the problem is two dimensional but lacks axial symmetry. However, there is an essential difference: The present self-similarity is for the dynamics, while the self-similarity discussed for the flow-induced air entrainment is for the steady-state flow approaching a transition. In fact, the universal scaling function for the shape in the previous study cannot be expressed as a Taylor series expansion but needs a Frobenius series with a singular exponent $3 / 2$.

We can further explain why $v_{0}$ is given by the second expression in Eq. (2). For this purpose, we note that $v_{0}$ is the magnitude of falling velocity $U\left(=d z_{G} / d t\right)$ of the disk in the oil phase, as seen from Eqs. (1) and (2). The falling velocity $U$ could be determined by the balance between the gravitational energy gain $\Delta \rho g R^{2} D_{0} U$ per time and an appropriate viscous dissipation, which should be the most dominant one among the following three [40]: dissipation associated with the Couette flow developed inside thin films between the disk surface and the cell wall $\simeq \eta(U / e)^{2} R^{2} e$ and dissipations associated with Poiseuille flows around the disk corresponding to the velocity gradient in the $y$ direction $\simeq \eta(U / D)^{2} R^{2} D$ and in the radial direction for the disk $\simeq \eta(U / R)^{2} R^{2} D$. The last dissipation may be smaller than the first two because $e, D \ll R$. However, the relative importance 
of the first and second is delicate; while the volume of dissipation for the first is well described by $2 \pi R^{2} D$, that for the second can be $\pi(c R)^{2} D$ with a fairly large numerical constant $c$. In the present case, $c$ seems indeed fairly large and the second dissipation seems the most dominant. This is because the balance of this dissipation and the gravitational energy gain gives $U \simeq \Delta \rho g D^{2} / \eta$, which is consistent with Eq. (2).

In summary, our theoretical result explains experimental findings, starting from the governing equations (6) and (7). By virtue of the Ansätze in Eq. (8), we showed that only a single length scale remains near the singularity and this length linearly scales with $t$. We further explained why we observed the self-similar shape dynamics demonstrated in Fig. 4 and represented by Eqs. (3) and (4).

\section{ACKNOWLEDGMENTS}

This work was partly supported by a Grant-in-Aid for Scientific Research (A) (No. 24244066) of JSPS, Japan, and by ImPACT Program of Council for Science, Technology and Innovation (Cabinet Office, Government of Japan) (Grant No. 2014-PM01-02-01).

[1] H. E. Huppert, Flow and instability of a viscous current down a slope, Nature (London) 300, 427 (1982).

[2] K. G. Wilson, The renormalization group and critical phenomena, Rev. Mod. Phys. 55, 583 (1983).

[3] P. Constantin, T. F. Dupont, R. E. Goldstein, L. P. Kadanoff, M. J. Shelley, and S.-M. Zhou, Droplet breakup in a model of the Hele-Shaw cell, Phys. Rev. E 47, 4169 (1993).

[4] J. Eggers, Universal Pinching of 3D Axisymmetric Free-Surface Flow, Phys. Rev. Lett. 71, 3458 (1993).

[5] R. E. Goldstein, A. I. Pesci, and M. J. Shelley, Topology Transitions and Singularities in Viscous Flows, Phys. Rev. Lett. 70, 3043 (1993).

[6] X. Shi, M. Brenner, and S. Nagel, A cascade of structure in a drop falling from a faucet, Science $\mathbf{2 6 5}, 219$ (1994).

[7] J. Eggers, Nonlinear dynamics and breakup of free-surface flows, Rev. Mod. Phys. 69, 865 (1997).

[8] J. Eggers, J. Lister, and H. Stone, Coalescence of liquid drops, J. Fluid Mech. 401, 293 (1999).

[9] D. G. A. L. Aarts, H. N. W. Lekkerkerker, H. Guo, G. H. Wegdam, and D. Bonn, Hydrodynamics of Droplet Coalescence, Phys. Rev. Lett. 95, 164503 (2005).

[10] J. C. Burton and P. Taborek, Role of Dimensionality and Axisymmetry in Fluid Pinch-Off and Coalescence, Phys. Rev. Lett. 98, 224502 (2007).

[11] J. D. Paulsen, J. C. Burton, S. R. Nagel, S. Appathurai, M. T. Harris, and O. A. Basaran, The inexorable resistance of inertia determines the initial regime of drop coalescence, Proc. Natl. Acad. Sci. USA 109, 6857 (2012).

[12] J. D. Paulsen, R. Carmigniani, A. Kannan, J. C. Burton, and S. R. Nagel, Coalescence of bubbles and drops in an outer fluid, Nat. Commun. 5, 3182 (2014).

[13] W. D. Ristenpart, J. C. Bird, A. Belmonte, F. Dollar, and H. A. Stone, Non-coalescence of oppositely charged drops, Nature (London) 461, 377 (2009).

[14] J. C. Burton, J. E. Rutledge, and P. Taborek, Fluid Pinch-Off Dynamics at Nanometer Length Scales, Phys. Rev. Lett. 92, 244505 (2004).

[15] J. C. Burton and P. Taborek, Bifurcation from Bubble to Droplet Behavior in Inviscid Pinch-Off, Phys. Rev. Lett. 101, 214502 (2008).

[16] D. Richard, C. Clanet, and D. Quéré, Surface phenomena: Contact time of a bouncing drop, Nature (London) 417, 811 (2002).

[17] K. Okumura, F. Chevy, D. Richard, D. Quéré, and C. Clanet, Water spring: A model for bouncing drops, Europhys. Lett. 62, 237 (2003).

[18] L. Xu, W. W. Zhang, and S. R. Nagel, Drop Splashing On a Dry Smooth Surface, Phys. Rev. Lett. 94, 184505 (2005). 
[19] J. C. Bird, R. Dhiman, H.-M. Kwon, and K. K. Varanasi, Reducing the contact time of a bouncing drop, Nature (London) 503, 385 (2013).

[20] Y. Liu, M. Andrew, J. Li, J. M. Yeomans, and Z. Wang, Symmetry breaking in drop bouncing on curved surfaces, Nat. Commun. 6, 10034 (2015).

[21] J. De Ruiter, R. Lagraauw, D. van den Ende, and F. Mugele, Wettability-independent bouncing on flat surfaces mediated by thin air films, Nat. Phys. 11, 48 (2015).

[22] P.-G. de Gennes, F. Brochard-Wyart, and D. Quéré, Gouttes, Bulles, Perles et Ondes, 2nd ed. (Belin, Paris, 2005).

[23] P. Doshi, I. Cohen, W. W. Zhang, M. Siegel, P. Howell, O. A. Basaran, and S. R. Nagel, Persistence of memory in drop breakup: The breakdown of universality, Science 302, 1185 (2003).

[24] L. Oddershede and S. R. Nagel, Singularity During the Onset of an Electrohydrodynamic Spout, Phys. Rev. Lett. 85, 1234 (2000).

[25] B. W. Zeff, B. Kleber, J. Fineberg, and D. P. Lathrop, Singularity dynamics in curvature collapse and jet eruption on a fluid surface, Nature (London) 403, 401 (2000).

[26] M. Yokota and K. Okumura, Dimensional crossover in the coalescence dynamics of viscous drops confined in between two plates, Proc. Natl. Acad. Sci. USA 108, 6395 (2011); in this issue, 108, 6337 (2011).

[27] J.-T. Jeong and H. Moffatt, Free-surface cusps associated with flow at low Reynolds number, J. Fluid Mech. 241, 1 (1992).

[28] J. Eggers, Air Entrainment through Free-Surface Cusps, Phys. Rev. Lett. 86, 4290 (2001).

[29] É. Lorenceau, F. Restagno, and D. Quéré, Fracture of a Viscous Liquid, Phys. Rev. Lett. 90, 184501 (2003).

[30] I. Cohen and S. R. Nagel, Scaling at the Selective Withdrawal Transition through a Tube Suspended above the Fluid Surface, Phys. Rev. Lett. 88, 074501 (2002).

[31] R. Burley, Mechanisms and mechanics of air entrainment in coating processes, Spec. Publ. R. Soc. Chem. 129, 94 (1993).

[32] I. Cohen, H. Li, J. L. Hougland, M. Mrksich, and S. R. Nagel, Using selective withdrawal to coat microparticles, Science 292, 265 (2001).

[33] A. Frohn and N. Roth, Dynamics of Droplets (Springer Science + Business Media, New York, 2000).

[34] K. G. Wilson, Feynman-Graph Expansion for Critical Exponents, Phys. Rev. Lett. 28, 548 (1972).

[35] J. Cardy, Scaling and Renormalization in Statistical Physics (Cambridge University Press, Cambridge, 1996).

[36] G. I. Barenblatt, Scaling, Self-Similarity, and Intermediate Asymptotics: Dimensional Analysis and Intermediate Asymptotics (Cambridge University Press, Cambridge, 1996), Vol. 14.

[37] Y. Chai, T. Salez, J. D. McGraw, M. Benzaquen, K. Dalnoki-Veress, E. Raphäel, and J. A. Forrest, A direct quantitative measure of surface mobility in a glassy polymer, Science 343, 994 (2014).

[38] See Supplemental Material at http://link.aps.org/supplemental/10.1103/PhysRevFluids.3.054004 for movies $1-4$.

[39] J. Eggers, Theory of drop formation, Phys. Fluids 7, 941 (1995).

[40] K. Okumura, Viscous dynamics of drops and bubbles in Hele-Shaw cells: Drainage, drag friction, coalescence, and bursting, Adv. Colloid Interface Sci. (2017), doi:10.1016/j.cis.2017.07.021. 